\title{
ESTUDO EXPERIMENTAL DE INCRUSTAÇÃO EM TROCADORES DE CALOR NO PROCESSO DE PASTEURIZAÇÃO DO LEITE
}

\author{
L. V. SIQUEIRA, C. G. C. C. GUTIERREZ \\ Universidade de São Paulo, Faculdade de Engenharia Química \\ E-mail para contato: caritocavero@usp.br
}

\begin{abstract}
RESUMO - O fenômeno de incrustação é um dos maiores problemas enfrentados pela indústria de alimentos devido à influência do material incrustado na diminuição da eficiência de troca térmica do equipamento durante os processos. Esse estudo aborda a influência e aporte das proteínas do leite na formação de incrustação durante o processo de pasteurização contínua por trocador de calor a placas. Foram realizados ensaios experimentais de pasteurização com soluções proteicas de Caseinato de Cálcio (Caseína) e Proteína Isolada do Soro do Leite (WPI) nas concentrações encontradas no leite integral $(20 \mathrm{~g} / 100 \mathrm{~g}$ de WPI e 80 $\mathrm{g} / 100 \mathrm{~g}$ de caseína). Foi medida a eficiência térmica do equipamento ao longo do processo e analisado o material incrustado sobre a superfície de troca térmica das placas. Durante o estudo, foram encontradas diferenças significativas na formação de incrustação entre as duas proteínas estudadas.
\end{abstract}

\section{INTRODUÇÃO}

$\mathrm{Na}$ atualidade, a qualidade e segurança microbiológica dos alimentos são uma das maiores preocupações dos consumidores e principalmente das indústrias de processos de alimentos o que induz a uma busca de alimentos mais saudáveis, com melhor qualidade nutricional e sensorial e longa vida de prateleira. Por esta razão, as indústrias tentam aprimorar cada vez mais os processos de tratamento de alimentos, criando-se assim a necessidade de melhorar o entendimento dos processos envolvidos na produção dos mesmos (Fito et al., 2007; Igual et al., 2010).

O tratamento térmico é uma das principais tecnologias aplicadas na indústria de alimentos para a preservação de alimentos e produção de produtos alimentos inócuos para o consumo, com vida de prateleira prolongada sem representar riscos para a saúde (Pereira; Vicente, 2010).

O fenômeno de incrustação na superfície dos trocadores de calor é um fator que também afeta o tratamento térmico de alimentos, como é o caso da pasteurização do leite (Boxler et $a l ., 2014)$. Este fenômeno é considerado como um dos problemas mais críticos na indústria de alimentos, pelo fato de ser necessária a parada do processo para a limpeza e remoção do material incrustado, não só para garantir as condições sanitárias do processo, mas também para garantir a boa eficiência térmica do equipamento (Petermeier et al., 2002). 
O processo de formação da incrustação é, em geral, de natureza complexa, e pode ser provocado por sedimentação, polimerização, cristalização, coqueamento, corrosão, ou por causas de natureza orgânica (Wallhäusser et al., 2012).

Atualmente há dificuldades na compreensão dos mecanismos de formação de incrustação do leite, devido precisamente à natureza complexa do leite. O estudo em questão visa melhorar a compreensão deste fenômeno presente nos processos de pasteurização.

O objetivo do trabalho é estudar o aporte das duas principais proteínas encontradas no leite integral (Caseinato de Cálcio e Proteína Isolada do Soro do Leite) na formação de incrustação durante o processo de pasteurização contínua com trocador de calor a placas.

\section{MATERIAIS E MÉTODOS}

Para o estudo experimental de incrustação, foram utilizadas as seguintes proteínas nas proporções encontradas no leite integral:

- Proteína Isolada de Soro de Leite 20 g/100 g (WPI) - 7,3 g/L - Arla Foods

- Caseinato de Cálcio 80 g/100 g (Caseína) - 29,1 g/L - Arla Foods

Para os testes experimentais de incrustação foi utilizado o pasteurizador de laboratório Armfield-43A provido de três trocadores de calor a placas (Figura 1). O equipamento e software adicional utilizado para a aquisição de dados foi o Compact DAQ-9172 e LabView 8.6 (National Instruments, USA), assim como um conjunto de 12 termopares de junta exposta inseridos nas tubulações do pasteurizador e posicionados nos pontos de medição mostrados na Figura 2.

Figura 1 - (a): Fotografia do pasteurizador de laboratório utilizado nos ensaios de incrustação; (b): Trocador de calor com placas identificadas.

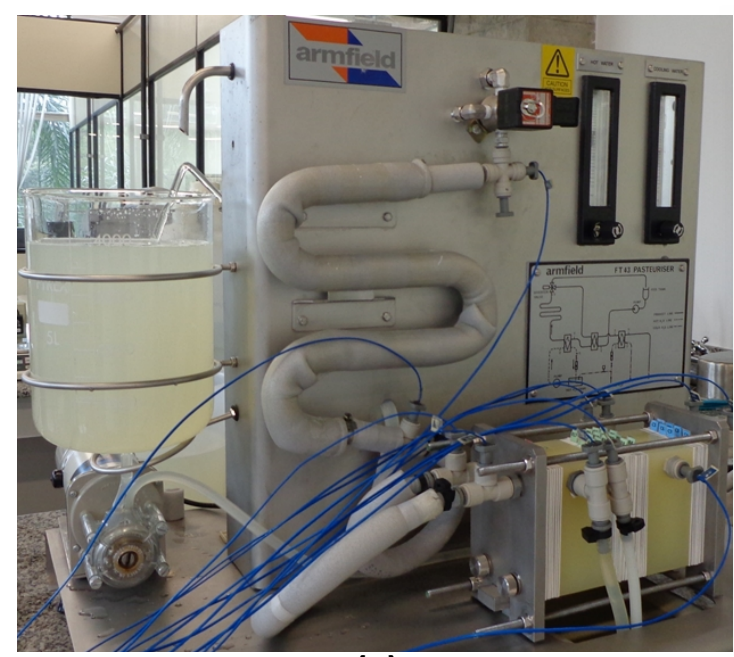

(a)

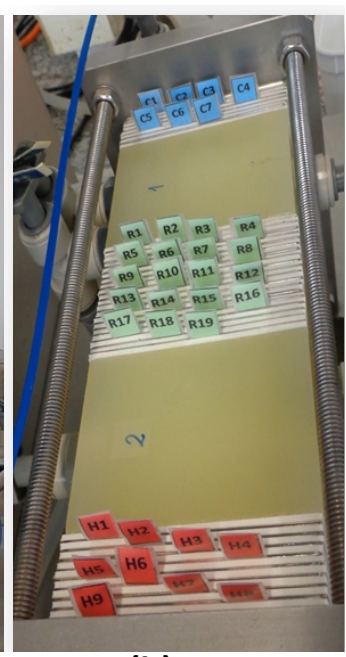

(b) 
Figura 2 - Esquema do pasteurizador com indicação dos pontos de medição de temperatura do equipamento (Gutierrez, 2013).

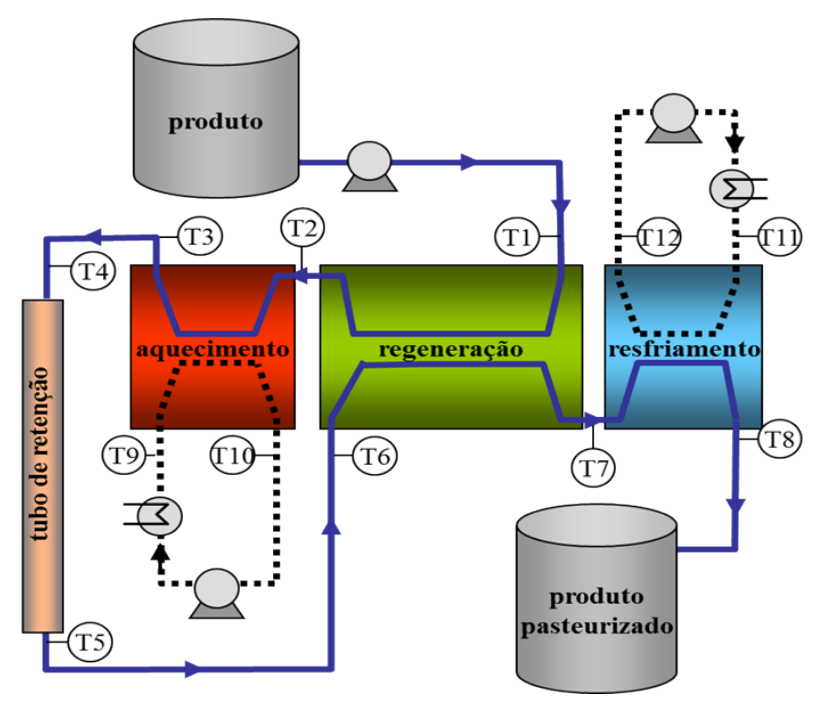

Os sistemas de aquecimento e resfriamento foram preenchidos com água destilada. Para cada ensaio experimental. O pasteurizador foi montado com as configurações do trocador estabelecidas para o ensaio (arranjo contra-corrente, um canal por passagem). Os ensaios foram realizados em malha aberta, sem controle de temperatura na saída do tubo de retenção. $\mathrm{O}$ controle de temperatura de alimentação do sistema de aquecimento foi fixado a $95^{\circ} \mathrm{C}$ com vazão de entrada de $60 \mathrm{~L} / \mathrm{h}$. A vazão de alimentação do produto foi de $20 \mathrm{~L} / \mathrm{h}$ e a temperatura de entrada de $23^{\circ} \mathrm{C}$. O sistema de resfriamento operou a vazão de $60 \mathrm{~L} / \mathrm{h}$ a temperatura aproximada de $4^{\circ} \mathrm{C}$.

Inicialmente, foi utilizada água como produto para estabilizar o equipamento e atingir o estado estacionário. Uma vez atingida a condição, foi dado início ao ensaio com a solução proteica, sendo registrados o tempo e temperaturas nos pontos de medição estabelecidos. Durante o processo, cuidados como a manutenção da temperatura de entrada do produto e do nível de altura no tanque de alimentação e o controle dos rotâmetros que medem a vazão dos sistemas de aquecimento e resfriamento foram necessários para garantir a estabilidade dos parâmetros de entrada do processo.

As placas do trocador de calor foram identificadas e pesadas antes e depois de cada ensaio para analisar e quantificar a massa incrustada após o processo de pasteurização. $\mathrm{O}$ material incrustado na superfície das placas foi seco em secador a vácuo a $60^{\circ} \mathrm{C}$ durante 6 horas aproximadamente para logo ser realizada a microscopia óptica do material incrustado.

\subsection{Tratamento de dados}

Para o cálculo do fluxo de Calor $(Q)$ trocado pela água e pelo leite foi utilizada a equação 1. A vazão mássica $(\dot{m})$ foi obtida experimentalmente.

$$
\dot{Q}=\dot{m} \cdot c_{p} \cdot \Delta T
$$


O poder calorífico $\left({ }^{c} p\right)$ foi calculado, para os canais com água, de acordo com a equação 2, e para os canais com solução proteica, de acordo com a equação 3 , onde ${ }^{x_{w}}$ é a fração mássica de água na solução (Cheng,Friis, 2007).

$$
\begin{aligned}
& c_{p(\text { água })}=5,2013 \cdot 10^{-7} \cdot T^{4}-2,1528 \cdot 10^{-4} \cdot T^{3}+4,1758 \cdot 10^{-2} \cdot T^{2}-2,6171 \cdot T+4226,1 \\
& c_{p(\text { leite })}=1675+2512 \chi_{w}
\end{aligned}
$$

A densidade do leite $\left(\rho_{(\text {leite })}\right)$ é calculada a partir da equação 4 , sendo ${ }_{f}$ a fração de gordura (Choi, Okac, 1986) e o cálculo da densidade da àgua ( $\left.\rho_{(\text {água })}\right)$ e realizada a partir da equação 5.

$$
\begin{aligned}
& \rho_{(\text {leite })}=1040,7-0,2665 \cdot T-0,0023 T^{2}-\chi_{f} \cdot\left(1,011+0,00976 T-4,81 \cdot 10^{-5} T^{2}\right) \\
& \rho_{(\text {água })}=2,08 \cdot 10^{-5} \cdot T^{3}-6,668 \cdot 10^{-3} \cdot T^{2}+4,675 \cdot 10^{-2} \cdot T+999,9
\end{aligned}
$$

Para calcular o coeficiente global de troca térmica $\left({ }^{U_{\text {exp }}}\right)$ foi utilizada a equação $6 . \mathrm{O}$ calor $(Q)$ foi obtido pela equação 1 e a Média Logarítmica das Temperaturas $\left({ }^{\Delta T}{ }_{l m}\right)$ foi calculada a partir da equação 7. A área da placa foi dada pela equação 8 , onde L é o comprimento da parte úmida da placa e $w$ é a largura da seção de troca térmica da placa.

$$
\begin{aligned}
& Q=U_{\text {exp }} \cdot A_{r e f} \cdot \Delta T_{l m} \\
& \Delta T_{l m}=\left(\frac{\Delta T_{2}-\Delta T_{1}}{\ln \frac{\Delta T_{2}}{\Delta T_{1}}}\right) \\
& A_{p}=w . L
\end{aligned}
$$

A Quantidade de Calor total foi dada pela média das Quantidade de Calor do leite e da água, como mostra a equação 9 .

$$
Q_{m}=\frac{Q_{\text {leite }}+Q_{\text {água }}}{2}
$$

\section{RESULTADOS E CONCLUSÕES}

Os resultados obtidos da taxa de calor transferido ao longo do processo na seção de aquecimento, onde são reportadas as maiores temperaturas do processo, para os ensaios com as duas soluções proteicas (WPI e Caseína) são apresentadas na figura 3 , junto às fotografias das placas incrustadas.

Embora a Caseína seja conhecida como uma proteína que precipita no meio ácido e WPI uma proteína que precipita a temperaturas maiores que $60^{\circ} \mathrm{C}$, foi possível observar maior precipitação por parte da caseína, como mostra a fotografia da figura 3. Em decorrência 
da maior incrustação, a redução de calor transferido ao longo do processo devido à formação de material incrustado foi mais acentuada para o caso da caseína o que indica o gráfico (a) da figura 3, que mostra a queda de transferência de calor ao longo do processo.

Figura 3 - Taxa de calor transferido (Q) ao longo do processo (a); fotografias das placas do trocador de calor incrustadas (b).

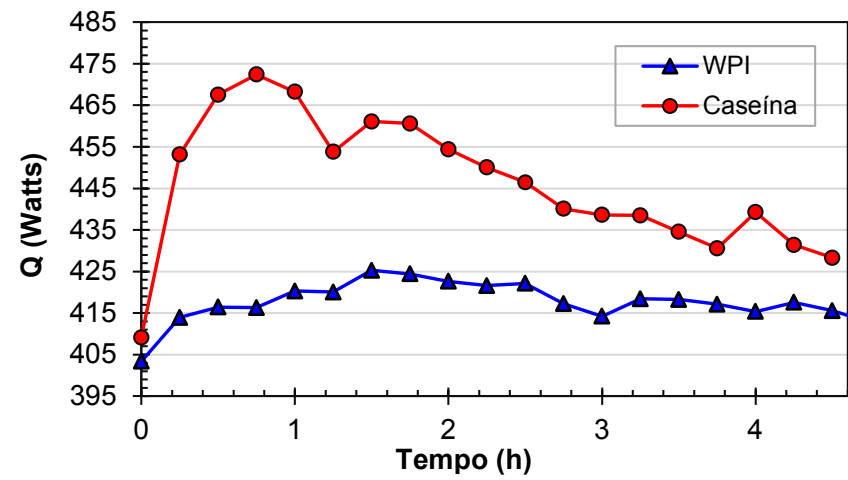

(a)

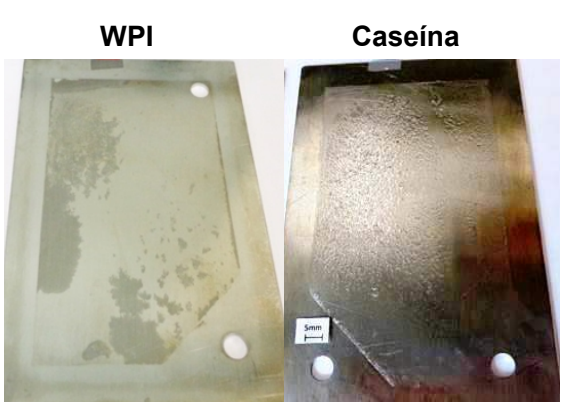

Placas do trocador de calor incrustadas

(b)

Os gráficos da figura 4 mostram a quantidade de massa incrustada na superfície de troca térmica das placas para cada solução proteína, indicando maior quantidade de massa incrustada no ensaio com a solução de Caseína. As setas nas barras dos gráficos na figura 4 indicam a direção do fluxo dentro do canal indicado, mostrando, na maioria das vesses, maior incrustação nos canais onde o fluido escoe na direção positiva do canal.

Figura 4 - Massa úmida incrustada nas placas após o ensaio de WPI e Caseína.
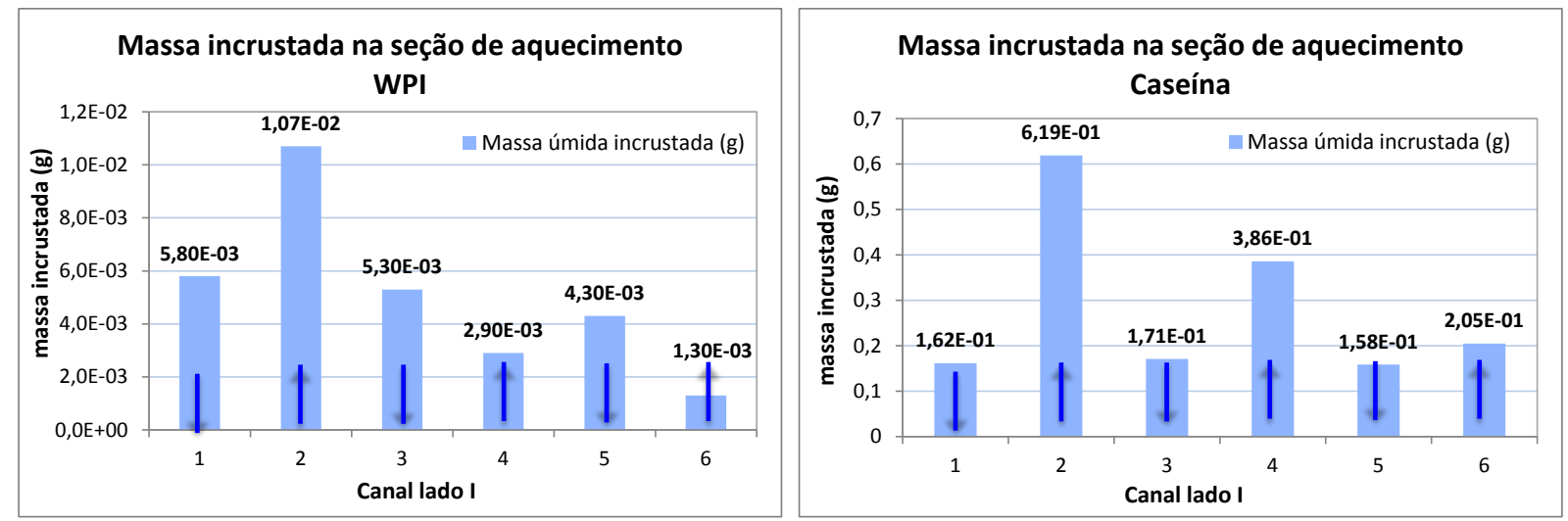

Foram encontradas diferenças significativas na estrutura do material incrustado das duas proteínas estudadas, sendo que no caso da caseína apresentou um aspecto gelificado e de fácil remoção (incompatível com a estrutura do material incrustado reportado para o leite), enquanto que no caso de WPI o material incrustado foi de difícil remoção e apresentou uma estrutura mais próxima da estrutura padrão de incrustação do leite integral reportada na 


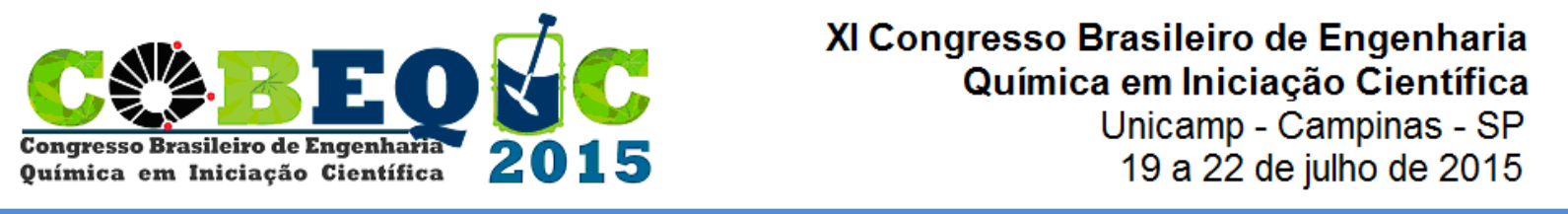

bibliografia por Bennet (2007), assim como uma semelhança entre as estruturas formadas pela massa incrustada, como mostra a Figura 5.

Figura 5 - (a): Micrografia da incrustação do leite integral (Bennet, 2007); (b):

Micrografia retirada no ensaio com WPI.

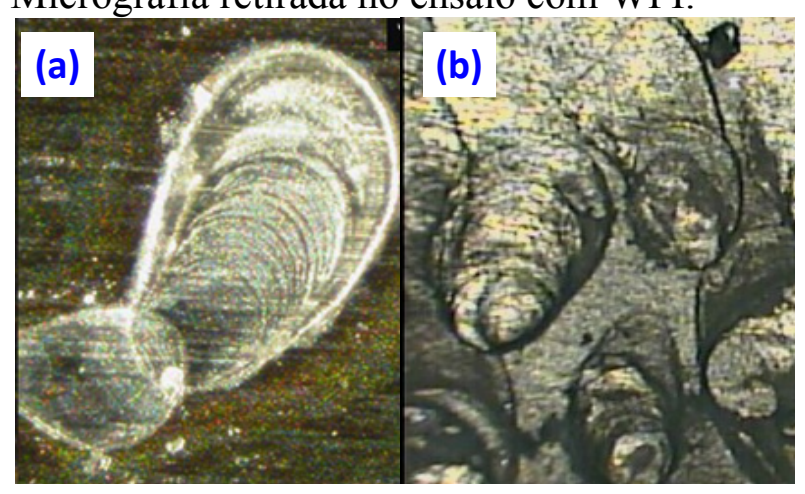

Sendo que o material incrustado com a solução proteica de Caseinato de Cálcio apresentou um aspecto gelificado e feito um teste de presença de amido na amostra o resultado foi negativo, indicando ausência de amido, são necessários estudos complementares para constatar se o comportamento da solução proteica de Caseinato de Cálcio, durante o ensaio de incrustação, possa ser atribuído especificamente ao processo de produção da amostra utilizada, que de alguma forma terminou influindo na composição final da mesma.

\section{REFERÊNCIAS BIBLIOGRÁFICAS}

BOXLER, C.; AUGUSTIN, W.; SCHOLL, S. Composition of milk fouling deposits in a plate heat exchanger under pulsed flow conditions. Journal of Food Engeneering, Elsevier, v. 121, p. 1-8, 2014.

CHENG, H.; FRIIS, A. Operability and Flexibility of Milk Production Line. Food and Bioproducts Processing, v. 85, n. C4, p. 372-380, 2007.

CHOI, Y.; OKOS, M. R. Thermal Properties of Liquid Foods - Review, In: OKOS, M. R. (Ed.). Physical and Chemical Properties of Food. St. Joseph: ASAE, 1986. p. 35-77.

FITO, P.; LEMAGUER, M.; BETORET, N.; FITO, P. J. Advanced food process engineering to model real foods and processes: The "SAFES" methodology. Journal of Food Engineering, v. 83, n. 2, p. 173-185, 2007.

GUTIERREZ, C.G.C. Análise dinâmica de um processo contínuo de pasteurização em trocadores de calor a placas. Tese (Doutorado) - Universidade de São Paulo, 2013.

IGUAL, M.; GARCÍA-MARTÍNEZ, E.; CAMACHO, M.M.; MARTÍNEZ-NAVARRETE, $\mathrm{N}$. Effect of thermal treatment and storage on the stability of organic acids and the functional value of grapefruit juice. Food Chemistry, v. 118; n. 2, p. 291-299, 2010.

PEREIRA, R.N.; VICENTE, A.A. Environmental impact $\mathrm{f}$ novel thermal and non-thermal technologies in food processing. Food Research International, Elsevier, v. 43, n. 7, p. 1936$1943,2010$. 
PETERMEIER, H.; BENNING, R.; DELGADO, A.; ZULOZIK, U.;HINRICHS, J.; BECKER, T. Hybrid model of the fouling process in tubular heat exchangers for the dairy industry. Journal of Food Engineering, v.55, p.9-17, 2002.

WALLHÄUSSER, E.; HUSSEIN, M.A.; BECKER, T. Detection methods of fouling in heat exchangers in the food industry. Food Control, Elsevier, v. 27, n. 1, p. 1-10, 2012. 